\title{
BMJ Open Towards safer healthcare: qualitative insights from a process view of organisational learning from failure
}

\author{
Negar Monazam Tabrizi (D) , ${ }^{1}$ Firas Masri ${ }^{2}$
}

To cite: Monazam Tabrizi N, Masri F. Towards safer healthcare: qualitative insights from a process view of organisational learning from failure. BMJ Open 2021;11:e048036. doi:10.1136/ bmjopen-2020-048036

- Prepublication history and additional supplemental material for this paper are available online. To view these files, please visit the journal online. (http://dx.doi.org/10.1136/ bmjopen-2020-048036).

Received 15 December 2020 Accepted 06 July 2021

Check for updates

(C) Author(s) (or their employer(s)) 2021. Re-use permitted under CC BY-NC. No commercial re-use. See rights and permissions. Published by BMJ.

${ }^{1}$ Global Development Institute, The University of Manchester, Manchester, UK

${ }^{2}$ Newcastle Business School, Northumbria University, Newcastle upon Tyne, UK

Correspondence to Dr Negar Monazam Tabrizi; negar.monazamtabrizi@ manchester.ac.uk

\section{ABSTRACT}

Objectives This study adopted a process view of organisational learning to investigate the barriers to effective organisational learning from medical errors. Methods Qualitative data were collected from 40 clinicians in high and low performing hospitals. The fit between the organisational learning process and sociotechnical factors was investigated systematically from a pre-reporting stage to reporting and post-reporting stages. Results The analysis uncovered that the major stumbling blocks to active learning lie largely in the post-reporting stages and that they are rooted in social rather than technical issues. Although the experience of the higherperforming hospital provides valuable pointers in terms of creating more trusting environment and using the potential of small failures towards ways in which the organisational learning process in the lower hospital might be improved, due to lack of local mangers' proactive engagement in integrating changes into practice the active learning takes place in neither of the hospitals.

Conclusions To ensure that the change solutions are firmly incorporated into the culture and routine practice of the hospital, we need to focus on fostering an organisational culture that encourages positive cooperation and mutual interactions between local managers and frontline clinicians. This process will lead to double-loop learning and an increase in system safety.

\section{INTRODUCTION}

Medical errors have long been a significant challenge and cause of mortality and morbidity in the healthcare industry. Evidence indicates that an estimated 42.7 million medical errors occur in hospitals every year, of which nearly $50 \%-83 \%$ could have been prevented. ${ }^{1}$ Severe harm, disability and death are the result of preventable medical errors ${ }^{1}$ with the associated cost of US $\$ 42$ billion annually. ${ }^{2}$

Given the importance of healthcare in patients' lives, not surprising, several studies have been conducted to understand the ways to develop a safer healthcare system. ${ }^{3-10}$ One of the more prevalent discussions within the literature on patient safety is around organisational learning and, crucially, the need for sharing experiential knowledge of medical errors. Learning from failures is a process

\section{Strengths and limitations of this study}

Forty interviews helped to provide a full and rich description of the socio-technical nature of the organisational learning process and systematically identify the barriers to effective organisational learning in each stage.

- Investigating the organisational learning process in high and low performing hospitals provided a compelling case for comparison to add to this area of research.

- Much of the insights on the strengths and weaknesses of organisational learning are gained from frontline clinicians.

- It would have been useful to know more about the viewpoints of the local managers.

- Overall, many of the studies on patient safety focused on developed countries and insights from the low-income and middle-income is lacking.

that can strongly improve organisational performance by developing new knowledge through finding errors and understanding their cause and effect. ${ }^{11-13}$ The suggestion here is that by exploiting knowledge of prior failure experience, correcting problems and replacing existing procedures, ${ }^{14}$ healthcare organisations can better avoid the preventable medical errors, thus reducing rates of future failure and decreasing failure-related costs, ${ }^{12} 15$ hence, minimising harm to patients.

It is evident that healthcare organisations are built on error management processes, ${ }^{9}$ and that much of the organisational learning is concerned with the sharing of medical errors. ${ }^{16}$ Sharing of medical errors, however, can flourish by developing an open, participative and blame-free culture, known as just culture ${ }^{17}$ For example, Vincent et $a l^{18}$ argue that clinicians are generally unwilling to be transparent about their medical errors due to fear of adverse consequences such as litigation and loss of job. They are not alone in this suggestion. Several scholars have suggested that medical professionals assume that sharing medical errors might constrain their 
career development. ${ }^{19-21}$ Just culture improves the level of trust and the quality of communication throughout the organisation as it encourages learning from failures rather than leading clinicians into getting into trouble or being criticised..$^{922}$

Subsequent literature, however, goes on to suggest that the healthcare industry is very complex and, therefore, successful learning from failures cannot only take place as a result of creating a just culture. For instance, the change of the culture requires all actors who have been involved in the learning process to share a common vision and this cannot occur without support from management. ${ }^{8}$ Since the healthcare industry is strongly hierarchical, the role of management is highly influential in staffing behaviours. ${ }^{23-25}$ Therefore, leaders need to facilitate the learning process by creating an environment where just culture is valued and considered as central to patient safety.

In addition to the cultural issues, information systems (IS) are a requirement for developing a practical approach to organisational learning from failures. ${ }^{8} 26$ IS are expected to enhance organisational learning by connecting individuals to share knowledge through shared storage ${ }^{27}$ and thus making the required information and knowledge available to authorised users more efficiently. ${ }^{27}{ }^{28}$ Given that effective communication and knowledge management are central in organisational learning, ${ }^{29}$ the learning process would be severely constrained in the absence of effective IS. Such systems provide professionals with an opportunity for sharing medical errors or safety concerns ${ }^{30}$ and enable managers to have access to the pool of information for extracting lessons, ${ }^{17}{ }^{31}$ thereby potentially improving health outcomes for patients.

The information that can be gleaned from these studies suggests that organisational learning from failures is a socio-technical approach. In other words, successful learning from failures depends on several elements, from soft issues such as cultural factors to hard issues such as technologies. It could be said that the interactions among these elements determine the learning results. Following this realisation, healthcare organisations such as the National Health Service (NHS) in the UK introduced a new service-wide system of knowledge management, which is the National Reporting and Learning System, ${ }^{32}$ as well as purposed-designed incident reporting IS to encourage organisational learning from failures. ${ }^{17}$ However, despite this, substantial changes have not yet been achieved in reducing the burden of patient harm due to preventable medical errors. ${ }^{2}{ }^{3}$ For example, 750 deaths a month are avoidable in the NHS of England. ${ }^{33}$ There is a case also for the USA where the number of preventable medical errors has risen to more than 250 000 people which makes medical errors the third largest cause of death in this country after cancer and obesity and more than guns and cars. ${ }^{34}$

These indicate that there are still barriers to effective organisational learning that they have not yet been fully understood. It is, therefore, time for a fundamental rethinking of the way that organisational learning from medical errors is investigated. Learning from medical errors has been treated as a black box by most researchers. This means that organisational learning from past failure experiences is seen as a single process, that is seen to constitute and act as a single entity; hence the details of preventing, analysing and learning are concealed. In this study, however, it is stressed that this cannot provide a complete and clear explanation of the barriers to effective organisational learning from failures. Therefore, this study proposes that to better understand the barriers to effective learning a process view of organisational learning, we need to emphasise the sequence of events that provides insights on the nature of the inner workings of the learning process from the pre-reporting stage to reporting, and postreporting stages. Dividing the learning process into different stages makes it possible to investigate each stage separately and therefore better understand where the learning process fails and, hence, can lead it in the best possible direction.

The process view of organisational learning has recently received attention and has been applied to understand the barriers to effective organisational learning in different industries. ${ }^{35-37}$ However, it is noticeably ignored in healthcare ${ }^{38}$ although exceptions exist. ${ }^{7}$ Nevertheless, Currie $e t$ $a l^{7}$ did not go beyond the identification and reporting of the incidents, and the details, especially on preventing the reoccurrence of the medical errors, are ignored.

The process view of organisational learning is developed based on the information processing perspective: acquiring, interpreting, distributing and storing information within organisations. ${ }^{29}$ Based on this foundation and following the Department of Health ${ }^{17}$ ['Department of Health sets the overall strategy, funds and oversees the health and care system in England' $],{ }^{39}$ the process of organisational learning from failure in this study is divided into the following four stages:

1. Initial incident identification.

2. Reporting incidents.

3. Deducing appropriate lessons from failures.

4. Incorporating changes into practice.

The first stage explains all the events that lead to the decision as to whether or not to share medical errors to alter organisational knowledge. In the second stage, clinicians document the actual incident report through IS. In the third stage, meaningful new knowledge should be then extracted from incidents reports. In the final stage, change solutions should be firmly incorporated into the culture and routine practice of the hospital and organisational behaviour should be altered as a result of changes made to organisational knowledge ${ }^{40}$ If all these stages were complete successfully, active learning will take place. ${ }^{35}$ This is the only way that healthcare organisations can be benefited from sharing failures. ${ }^{17} 41$ This highlights the paramount importance of the achievement of sustained improvements in each of these stages since a 
flaw or defect in one stage can prevent active learning from taking place.

So far, it is clear that successful learning from failures is a series of stages and must consider various elements to ensure a positive outcome. It is proposed that the learning process is influenced by the impact of sociotechnical factors (eg, organisational culture and technology support). As a result, the successful learning process cannot occur without the support of sociotechnical factors. Similarly, the impact of socio-technical factors on successful learning from failures cannot be investigated in detail without considering the different stages of the learning process. Accordingly, the fit between the learning process and socio-technical factors must be emphasised to investigate the challenges to active learning from failures.

Hence, this research investigates the process of organisational learning from medical errors with the aim to provide a rich description of its socio-technical nature. Such a description enables us to unpick factors and conditions necessary to promote and sustain each stage of the learning process, which in turn can reveal how and why effective and active learning can take place. The main focus of this research falls within the context of NHS of England, where, as discussed earlier, there are still challenges in providing safe and high-quality care.

The section on methods explains how the two NHS hospitals and the 40 clinicians interviewed were selected. It also explains the strategies for data collection and analysis. This is followed by an in-depth, socio-technical analysis of the organisational learning process in the NHS. The discussion highlights the findings and calls attention to the need for a focus on enhancing post-reporting stages and fostering an organisational culture that encourages positive cooperation and mutual interactions between local managers and frontline clinicians.

\section{METHODS}

To be able to gain insight into the focus of this research, a full and rich description of the phenomenon was needed. In doing so, the process of learning from medical errors is compared in two NHS hospitals in England with different performance levels so that to be able to (1) understand if the learning process differs in high and low performing hospitals, (2) identify the dimensions where one is better than the other and (3) understand the main reasons for the differences as well as similarities between the learning processes. The two case studies were similar in terms of staffing level and the incident reporting system. However, they were strategically chosen to provide comparative insights, with one being among the top 10 high performing NHS hospitals in England, while the other was categorised among the 20 lowest performing NHS hospitals. This helps to provide not only richness in variety but also yields opportunities for literal replication across the two cases. ${ }^{42}$ For confidentiality, and to protect the identity of the chosen NHS hospitals, the hospitals are identified by codes rather than names. For example, in the first case study, the hospital which is considered as high performing is coded as hospital A and in the second case study, the hospital which is considered as low performing is coded as hospital B.

Data were collected over a 9-month period. Semistructured interviews were carried out for data collection. The sampling focus was based on actors involved in the process of learning from medical errors that deeply understand the situation. We were, in particular, looking for those clinicians who work closely in a team so that to comprehensively explore the interactions among the learning process actors. Therefore, when considering these reasons, we decided to interview clinicians who worked in the same ward. Considering the busy environment of the healthcare industry, two similar wards in each case study were targeted. In total, 40 (10 in each ward and therefore 20 in each case study) interviews were undertaken across two hospitals, with an average of $60 \mathrm{~min}$ each. Interviews were conducted in the workplace and the sample was selected based on the interest and availability of the healthcare providers. With permission received from the ward managers, clinicians were approached face-to-face by the field researcher for recruitment in each ward. Care was taken to select participants representing different positions and roles in each ward. The set of interviewees included doctors, nurses, physiologists and dieticians. An overview of participants by occupation, gender and work experience is presented in table 1. Potential identifiers were removed to preserve participants' anonymity. The key themes discussed during interviews aimed at understanding the factors that drive each stage of the learning process or cause the deeprooted barriers in each stage from the frontline clinicians' perspective.

In the process of interview data collection, we followed the principles suggested by Saunders $e t$ at $t^{43}$ to guarantee the reliability and validity of our research. Faceto-face interactions and prolonged involvement in the field were seen as a powerful means to build trust and

\begin{tabular}{|c|c|c|c|}
\hline & Hospital A & Hospital B & Total \\
\hline \multicolumn{4}{|l|}{ Occupation: } \\
\hline Nurse & 8 & 8 & 16 \\
\hline Doctor & 7 & 8 & 15 \\
\hline Dietician & 2 & 2 & 4 \\
\hline Physiologist & 3 & 2 & 5 \\
\hline \multicolumn{4}{|l|}{ Gender: } \\
\hline Female & 9 & 13 & 22 \\
\hline Male & 11 & 7 & 18 \\
\hline \multicolumn{4}{|l|}{ Years in the hospital: } \\
\hline More than 10 years & 6 & 5 & 11 \\
\hline Between 5 and 10 years & 9 & 8 & 17 \\
\hline Between 1 and 5 years & 5 & 7 & 12 \\
\hline
\end{tabular}


rapport between the field researcher and clinicians. This helped to collect sufficient data and recruit new participants into the study during data analysis until a saturation point was reached when no new information emerged from the data. Semi-structured interviews leave room for unpredictable questions but maintain consistency within the structure. ${ }^{44}$ The field researcher followed the interview guide (see the online supplemental file 1), which was developed following Saunders $e t a l \mathrm{~s}^{43}$ guideline, to ensure all participants in the selected hospitals get the same experience and thus diminishing sources of bias. It is recognised that interviewees could have been reluctant to discuss their mistakes for the fear of managers' criticisms. Interviewees were given a participant information sheet, providing key information about the study including data confidentiality and anonymity. During the interviews, words were carefully chosen, and an indirect questioning technique was used to minimise socially desirable bias. ${ }^{45}$ Since the researcher was likely to use the new knowledge ineffectively at first (eg, false interpretation), she applied the mirroring question and answer technique. ${ }^{46}$ If necessary, the researcher took the words or phrases used by clinicians and repeated them in a subsequent question in order to minimise false interpretation. The findings of the study were also checked with three clinicians in each hospital.

All the interviews conducted for this study were taperecorded and then transcribed for in-depth analysis. To analyse the interview data, deductive thematic analysis was employed, which required the researcher to produce a list of codes that represented the themes identified in the textual data. ${ }^{47}$ The field researcher undertook the initial coding and produced a list of codes that represent the learning process, that is, initial incident identification, reporting incidents, deducing appropriate lessons from failures and incorporating changes into practice. These themes were used to identify the factors that have an impact on the learning process. All elements of data were added and categorised by the relevant themes in a spreadsheet. The second researcher then coded samples of data to test the consistency of codes. Coded interviews were discussed by the researchers and differences of interpretation were discussed until consensus was reached. Finally, the coded data were regularly compared and contrasted to interpret the views of individual respondents.

\section{Patient and public involvement}

Patients and/or public were not involved in the study.

\section{The process of organisational learning from past failure experiences in the NHS \\ Initial incident identification}

When an incident happens, clinicians who are involved in the incident need to decide whether or not to report it. In doing this, actors have to be adequately motivated; otherwise, they will not be willing to contribute. ${ }^{40}$ From our fieldwork, it was evident that clinicians in both hospitals were willing to report the incident and start the learning process. An overwhelming majority of clinicians in both hospitals believed that learning lessons from mistakes could develop better practices in the future and consequently improve patient safety. As a result, patient safety promotes a favourable attitude towards reporting medical errors and, thus, is motivating.

Clinicians here are eager to provide high-quality services to patients and look after them in the best possible way. So, it's more safety of the patients, and if I bring something everybody gets to know it and next time try to prevent it (Doctor 11, Hospital A).

I would think about patient care all the time. That's why I did nursing and patient care. So, it's always the patient. It could be awkward after [incident reporting], I feel bad, but that's this job (Nurse 22, Hospital B).

However, despite these, fundamental differences in the culture of the two NHS hospitals involved in this study were found. As already highlighted, the key to learning from incidents is to create an organisational climate in which clinicians psychologically feel comfortable to report their errors. ${ }^{48}$ In the case of hospital A, almost all clinicians confirmed that the hospital exposes clinicians to an appropriate culture of blame-free assessment and learning which does not lead clinicians into getting into trouble or being criticised.

I don't take it [incident] as something to penalise one. It's all about tracking up something where everyone can learn. We do a lot of these [incident reporting], and it's all about preventing the same thing happen again (Dietician 3, Hospital A).

Conversely, evidence indicates that the culture of hospital B errs towards a blame culture. However, the interesting point here is that while the existing organisational culture within the hospital can sometimes constitute a barrier to reporting the incidents, most of the time it affects the quality of the incidents reports. Interviewees repeatedly expressed that since they work in a team, when an incident happens, especially a severe incident, everyone will know about that. Therefore, they do not have a choice not to report it. In effect, clinicians in this hospital might report the incident, however, because of the lack of trust in the culture, they sometimes act against the identification of the true causes of the failure for fear of punishment and appearing as incompetent. This is an important issue as the accuracy of the incident report is very important in identifying the real causes of the incident and incorporating the necessary changes into practice.

In theory, it shouldn't be a blaming culture, but in real life, it feels like that isn't the case. So, I think the cultural thing isn't quite right and [they're] trying to improve that but there is a long way to go. But there seems to be this trend, or it's a cultural thing that we need to find out something that went wrong 
and then it can be addressed. And sometimes you just have a scenario which is just bad luck, everything was done to good practice and it was just bad luck and there was nothing that could have been done to avoid it. But people investigate these things that we have to come up with some recommendations and that's quite off-putting (Consultant 21, Hospital B).

If I understand correctly how it works, an incident report is basically that you don't get blamed for it, you don't get into trouble over it. They offer you training if you need training and then you try to learn something for your organisation. Whereas often I find in the NHS, you get blamed; find some blaming news, you know, is to blame who is responsible and that's the opposite of helpful (Nurse 25, Hospital B).

The findings of the case study revealed that mutual trust between ward managers and clinicians could alleviate the negative impact of the blaming culture in this hospital and increases the likelihood of successful incident reporting. In other words, when ward managers are perceived as trustworthy, then incident reporting and accuracy of the reports are more likely. This is because clinicians are certain about the intention of their senior management and do not fear job security.

If you make a mistake like a drug error then you might not want to fill in the form-"oh it can cause a matter to me". But you might want to check with the ward manager and see what they suggest. Here our ward manager ... is really supportive. You know, the managers should be quite supportive then people can trust and be honest about what happened (Nurse 30, Hospital B).

\section{Reporting incidents}

At this stage, clinicians prepare the actual incident report by documenting who was involved in the incident and what the situation was. As documented elsewhere, ${ }^{7}$ the incidents reports are first received by local managers and then by the National Patient Safety Agency. IS is a particularly important part. This study found that a unified mechanism and purpose-designed systems of incident information gathering are in place in both hospitals. As a result, incidents can be easily documented and hence the lessons can be identified accordingly. The findings of the case study revealed that incident reporting at both hospitals is all based on information communication technologies (ICTs). Since technology is involved, technical support is also required so that to protect the use of ICTs for the purpose of incidents reporting. Clinicians in both hospitals confirmed that the online system matches their requirements and that they have the required skills and knowledge to use the system. One of the physiologists in hospital B commented on the value of this electronic system as follows:

There used to be a huge booklet everyone saw as a drag of timing. But now it's straightforward, you go along and fill out the boxes. ... the good thing about that [online forms] is, if you don't fill in a section, it tells you "you cannot move on". So, they [management team] like that because all the information is given whereas, if it is on paper, it's easy to skip the sections.

In light of the above statement and similar comments from other interviewees, NHS hospitals recently switched from a paper-based system to an electronic system for reporting incidents. Most of the clinicians confirmed that the new electronic system is more effective in several ways. First, it helps in transferring a complete report that is required by the management team. Second, managers can access the report instantly and extract valuable lessons. Third, departments with more and recurring incidents can be detected. Fourth, the system provides the opportunity for confidential reporting.

However, this system is not without a problem. When clinicians fill out the online form and send it, there is no chance of modifying the form if necessary. Whereas, with the old paper-based system, clinicians could add more information if they could remember something new. It is also common sense that clinicians need to have access to computers so that to report the incident. There are few numbers of personal computers available on each ward, which are shared by clinicians, and like the findings of Sujan, ${ }^{49}$ this seemed to retard the incident reporting.

So, certain hours through the day you've got just a massive volume of staff, all want a computer. Sometimes you might need a computer and stand free, and you do what you want because everyone is doing treatment sessions. But at certain hours of the day, it isn't easy to get computers (Nurse 8, Hospital A).

\section{Deducing appropriate lessons from incidents reports}

Once incidents have been reported, they must be properly analysed by the management team. They use the reports to deduce lessons and decide on actions which need to be taken. These actions depend on the nature of the incident, which would show how serious it is. For example, if there was a severe incident that happened because of the clinicians' lack of knowledge and skill, the management team would send the clinicians to relevant training. All evidence suggested that the management team in hospital A actively deal with the incident reported regardless of being a small or large failure. They provide the clinicians involved in the incident with feedback and deduce the lessons from the pool of available incident information/knowledge through analysis.

However, in the case of hospital B, while incidents are often identified and reported, clinicians perceived that little effort is made from the management side to deduce lessons from some potentially important streams of incidents reports. Clinicians in hospital B overwhelmingly said that when they report the incident most of the time 
there is no feedback afterwards unless for a highly visible crisis.

The trouble I have with the system is they [management team] never give feedback. So, if you send it off, it seems to go somewhere, but you have no idea what happens next. That's very annoying because you're putting it in because you think something is wrongyou want at least feedback (Dietician 37, Hospital B).

So, that's almost us making the people above us aware that this thing [incident] happened. But then I think the worst thing is when you send the form, you don't know what happens to that. We don't get to find out what the consequences were because of that. For example, patients are transferred from another ward when they are sick, you know, they should have to tell us [their details]. If we fill out the [incident] form, does that ward find out that we filled the form out? Do they know that what they did was wrong? I don't think so, because I know the wards here. I don't think I've ever heard if anybody said that there was a report about us as a ward or anything (Nurse 29, Hospital B).

We fill out forms, but that's really annoying because we don't know what's happening with the form we filled out. I think it would be quite nice to know what the outcome was. And I want to know something has been done (Doctor 31, Hospital B).

The major issue raised here is around the need for visible management support. While managers in hospital A highly support the learning process by adequately analysing the reported incidents, whether small or large and providing feedback to clinicians making reports, managers in hospital B do not normally support the process as such and largely ignore the small incidents. This means that at hospital B, most of the time, the learning process is stopped at this stage due to the lack of management support.

\section{Incorporating changes into practice}

Once sound solutions have been derived, the management team should put the lessons into practice so that to ensure that the essence of the learning points is captured by all relevant actors. ${ }^{17}$ Then, all the actors should integrate the useful part of the knowledge into their knowledge and apply it into their day-to-day job. Following this, the management team need to make sure that organisational behaviour is, in fact, changed as a result of the lessons deduced from incidents reports. This requires continuous monitoring to make sure that new action plans are implemented and followed by clinicians. This is important because there might be barriers in place in the use of new procedures that need to be solved and rectified; otherwise, the true active learning process will fail.

This study found that the identified lessons in both hospitals are shared with frontline clinicians through passive communications. Managers hand decisions down through the chain of instructions/procedures and largely neglect supporting the frontline clinicians in the use of new knowledge. This means that, when any new procedures are put into practice, the management team usually do not ensure whether or not they are being used effectively by clinicians. As documented elsewhere, ${ }^{5}$ clinicians are less likely to be able to use the knowledge and learn effectively through passive interactions.

There are sometimes when we report things, but they didn't get acted on, how we like. We see the same problems happen over and over again. But if you report something, you would not want to see it again (Nurse 18, Hospital A).

I know once the patient was affected due to NG. The procedure changed about how you have to monitor, how far the tube was in, you have to measure the tube every time before you feed, make sure that the tube is in the right place and things like that. So, another procedure changed for that, for instance, putting there, it was sort of like read it and sign it. So, nobody said to me have you read that and sign that nobody checks to see if I've read and signed (Nurse 32, Hospital B).

The interview data, however, appeared to indicate that the major incidents are discussed in staff meetings and managers try to highlight the key points. Additionally, when necessary, the management team, due to patient safety, see a benefit in helping resolve the issue.

We do have staff meetings and if there is something very serious or have a major risk associated with it or a patient death or something, the management would feedback to the staff (Doctor 1, Hospital A).

However, it is argued that if organisations focus on a problem for a short period of time and forget about it when key stakeholders move on or new priorities emerge, effective learning does not take place. ${ }^{17}$ Evidence from both hospitals suggested this is peer support that plays a more significant role in continuous monitoring of improvements and changes in practice.

We've had an incident where the patient fainted because the feeding tube was in the wrong place. So, now the procedure is so strict. So, that's such a serious incident. That's why I think it was feedback formally and also informally through ward members of staff being like "oh my god do you know this happened". And then now everybody knows about the incident. But this is more the nurses that helped each other to do the right thing. For instance, if somebody is not doing it correctly, one of the nurses says "you need to do this, it's important because we had an incident where a patient fainted" (Nurse 24, Hospital B).

A patient came to the hospital with 5 different medications but their GP [General Practitioner] had prescribed 10 different ones. We kept giving them the 5 that they brought and missing 5 other tablets and 
the patient died because of that. Now when new patients are admitted, the first thing that our pharmacists say to you [nurses] is about their medications and emphasize their importance because a patient died. Pharmacists have a system that they can log onto to check what GPs prescribed. But there is nothing on the system to say patients have died because of this. It's just passed on through their [pharmacists] knowledge and their understanding of it (Nurse 11, Hospital A).

As these quotes demonstrate, the success of organisational learning and service improvement also lies in informal learning in the workplace. Some of the clinicians, however, were particularly concerned that sometimes peers understand the procedures differently and therefore provide feedback in different versions. Also, some of the clinicians remained sceptical about whether they can provide feedback to their peers.

One person says "oh I was told you must do like this" and then some else says "I was told you must do that". And then you worried oh one person wants me to do that and then another, you know. I feel like not everybody read from the same book (Nurse 22, Hospital B).

It is dangerous because I could know something then that's like it's not really my place then to tell other people because I'm not the ward manager. But because I've chosen to go out there and ask the questions and get the answers should it be then down to me to feed that back (Nurse 18, Hospital A).

Overall, the analysis demonstrates, on the one hand, peer support and informal learning can help to promote organisational learning and service improvement, but it also reinforces the role of managers to check clinicians' understanding of the new procedures and play a more active role in the monitoring of improvements and changes in practice. The findings suggest that managers' lack of proactive engagement in supporting frontline clinicians in the use of new procedures resulted in the reoccurrence of the same incidents, confirming that active learning did not take place in either of these hospitals.

\section{DISCUSSION AND CONCLUSION}

This study theorised treating organisational learning as a black box cannot provide a comprehensive explanation of the barriers to learning from past failure experiences. It thus argued that to understand how to make healthcare safer, we need a focus on a process view emphasising the sequence of events that provides insights on the nature of the inner workings of the learning process. Based on a sample of 40 interviews from two NHS hospitals, this study systematically revealed the main barriers to active organisational learning. The learning process was investigated from the stage of pre-reporting medical errors (namely, the stage of initial incident identification) to the stages of reporting and of post-reporting incidents (namely, the stage of deducing appropriate lessons from failures and that of incorporating changes into practice).

The comparison picture of the learning process in hospital A (a high performing hospital) and hospital B (a low performing hospital) is presented in table 2. Colours are used in this table as signifiers to represent the robustness or otherwise of each stage of the learning process in each of the two hospitals. In specific terms, green columns represent the areas with fewer issues and amber columns indicate the areas that need significant improvement. The socio-technical interplay among soft and hard issues that describes the strengths and weaknesses of each of the stages in the two hospitals indicates that there are significant differences as well as similarities between the two hospitals. Although hospital B does not compare well with the learning process in hospital A, there is one thing in common: the ultimate goal of the learning process, which is preventing the reoccurrence of the same incidents, is not achieved, regardless of the overall NHS classification whether as high or low performing healthcare organisations. The analysis uncovered that the major stumbling blocks to active learning are mainly rooted in

Table 2 Comparison of learning process between hospital A and B

\section{Stages of organisational} learning from medical errors Hospital A

1. Initial incident identification

\section{Reporting incidents}

3. Deducing appropriate lessons from incidents reports

4. Incorporating changes into practice practice.

\section{Hospital B}

Patient safety highlights the importance of incident reporting. However, the majority of interviewees (15/20) confirmed that a blaming culture can impact the accuracy of the incident reports as clinicians do not always feel psychologically safe to reveal the true causes of the failure.
Managers only support large failures and hugely ignore small incidents and do not actively provide feedback to clinicians.

Passive communication and lack of managers' proactive support and engagement in integrating changes into 
the post-reporting stage (ie, incorporating changes into practice) and ultimately managers wishing to make the learning process a success need to pay more attention to social issues.

The findings of this study point out to the fact that clinicians from all professional groups acknowledge the importance of reporting medical errors including near misses in increasing patient safety, and therefore, are willing to report their mistakes with an aim to contribute to organisational learning and patient safety. This supporting attitude seems to have been developed more recently as the previous studies indicated that medical errors are normalised within the medical context to the extent that clinicians, ${ }^{721}$ especially doctors, perceive incident reporting as unnecessary. This is a positive change as the learning process cannot trigger in the absence of clinicians' willingness to report their mistakes. In recent years, there has been more emphasis on patient safety and alignment of incident reporting to better care for patients which, in turn, improved a more favourable attitude to the importance of reporting incidents.

Although the online incident reporting system in both hospitals has some limitations (eg, recurrent lack of access to a computer, lack of opportunity to add more information by clinicians after submitting the online form), its benefits outweigh its limitations. The findings indicated that the newly introduced online IS makes information sharing between the clinicians and the management team more effective and efficient. The findings also confirmed that clinicians found the use of standardised forms useful in documenting the incidents. This argument is in contrast with the conclusion drawn by Currie $e t a l^{7}$ who stated that the purpose of the standardised forms and the information that they intended to collate are not well understood by the clinicians. The newly introduced electronic system may explain this contrast. The findings of this study, however, are in line with previous findings of ${ }^{7}$ in that the managers do not always trust the accuracy of the reports. Nonetheless, this issue is rooted in the hospitals' culture. Reflecting on Hignett $e t a r^{3}{ }^{3}$ analysis of NHS culture, the just culture and clinicians' fear of being blamed because of their mistakes remain an issue in some NHS hospitals. The findings of this study show that, unlike hospital A, just culture is not yet well established in hospital B and this negatively affects the learning process from the very first stage (ie, initial incident identification) as clinicians do not always feel psychologically safe to reveal the true causes of the failure. The findings further indicated that when the local managers are perceived as trustworthy, the chance of reporting/documenting more accurate reports will increase, hence the importance of the role played by local managers in the organisational learning process and patient safety.

The challenge, however, is that the local managers do not always support the learning process which constitutes a barrier to the active organisational learning from failure experiences. In hospital B, the lack of management support is more visible and, therefore, the learning process is normally interrupted in hospital B earlier than in hospital A. While clinicians report their mistakes intending to receive feedback from the management team, the local managers in hospital B normally do not provide feedback unless for a highly visible crisis. However, 'every failure is a learning opportunity' and, therefore, learning from small failures or near misses can be as important as learning from large failures in preventing future occurrence of errors ${ }^{41}$ This lack of management support leads to missing the opportunity to learn from small failure cases as well as sending the wrong signal to frontline clinicians that reporting medical errors is not essential. ${ }^{49}$ In hospital A, however, managers support the organisational learning process more actively by providing feedback to the clinicians and making better use of the potential of the pool of information to identify the lessons from both small and large failures. Such support in hospital A helps the lessons at least being more actively learnt in their immediate environment by those reporting the incident.

Despite this, the main issue that both hospitals share come from incorporating the lessons into practice and spreading the lessons learnt beyond their immediate environment. The management team do not actively collaborate with clinicians to incorporate knowledge into practice, but knowledge is shared through passive communications. The finding reflects Sujan's ${ }^{49}$ perspective that informal learning through peer support and informal discussions on the wards can help in implementing changes after incidents. However, as with Adler's ${ }^{50}$ findings, frontline clinicians lack the required incentives to use the new instructions and procedures effectively. Equally, frontline clinicians may think they do not have the power/authority to monitor changes in practice by providing feedback to their peers. Thus, more support from managers is needed to monitor and ensure that new action plans are implemented and followed by clinicians. However, managers' lack of proactive support and engagement in incorporating changes into practice resulted in dispersion and disconnection between the ideal standard and practice. ${ }^{5}$ In effect, the same mistakes repeatedly happen confirming that active learning does not take place and the attempt to alert the organisational behaviour is hampered by the lack of local managers' support.

The present study contributes to the field of safety management system by detecting which factors are encouraging/impeding the learning process in what stage and why from clinicians' perspective. In effect, the study argues that to enhance organisational learning and make healthcare safer, it is crucial to pay more considerable attention to local leadership and post-reporting stages. Although the NHS policies remain the same for all NHS hospitals, significant differences (eg, just culture vs blaming culture; extracting lessons from both small and large failures vs ignoring small failures) were observed in the learning process of the two NHS hospitals investigated in this study where these differences can be traced back to the local mangers attitude and behaviour. Additionally, 
and interestingly, the main reason for the ineffective organisational learning (namely, the reoccurrence of the same mistakes) in both hospitals is also rooted in the lack of proactive engagement of the local managers in incorporating changes into practice. This is where the learning process often seems to fracture and therefore the mistakes reoccur. It makes sense to suggest then a focus on promoting a collaborative culture among local managers is crucial given that for organisational learning to be effective, local managers need to work collaboratively with frontline clinicians to reduce the gap between the recommended procedures and practice and improve the patient safety. Such collaboration will encourage double-loop learning ${ }^{51}$ where local managers and frontline clinicians can work together to identify the problems, correct them and, more importantly, ensure whether the new procedures are performed and are effective.

In conclusion, the formal incident reporting system may seem ineffective ${ }^{49}$; however, it is essential in improving patient safety. A formal incident reporting system facilitates communicating explicit knowledge from people in higher ranks and spreading the critical lessons from their immediate environment. Nevertheless, the success of this system primarily lies in the local managers' support and collaboration with frontline clinicians. Promoting informal learning and giving more power to clinicians to monitor changes on the wards can complete the formal organisational learning process. However, managers will need to encourage frontline clinicians to use the new procedures correctly and effectively and address any knowledge gap and misunderstanding of the frontline clinicians in the use of new procedures. Institutionalising social interaction and cooperation between managers and frontline clinicians contribute to change implementation $^{52}$ and alerting organisational behaviour. These findings could also have important implications for private hospitals, especially given studies show that private hospitals are not more accountable, efficient and medically effective than public hospitals. ${ }^{53}$

Being retrospective, our study has a certain number of research limitations. First, the results of this study indicate that there are differences in the organisational learning process in a high and low performing hospital where these differences are mostly rooted in a trusted working climate and personal commitment. However, more quantitative analysis is needed to examine whether there is a connection between the quality of organisational learning and the hospital's overall performance. Second, the aim of this study was to investigate how the organisational learning process can be improved from frontline clinicians' perspective. Thus, the recommendations of this study for promoting the learning process in general and for local managers in particular are only informed by the beliefs and perceptions of the clinicians. However, given the active role of local managers in the learning process and organisational change, more research is needed to bring more insights from the perspective of local managers. Thirdly, the scope of this research is limited to western healthcare organisations (ie, NHS in England) where safety measures have been applied. A future research agenda needs to engage in more empirical and conceptual clarifications of some of the issues raised here in low-income and middle-income countries where safety measures have not yet been adapted for successful application. ${ }^{54}$

Contributors NMT was involved in data collection and analysis and writing the manuscript. FM reviewed the data analysis and the final draft.

Funding The authors have not declared a specific grant for this research from any funding agency in the public, commercial or not-for-profit sectors.

Competing interests None declared.

Patient consent for publication Not required.

Ethics approval The ethical approval for this study was gained from the NHS Research Ethics Service (Ref. 13/WA/0138), and the research passport for each participating hospital was received from the local hospital's R\&D department.

Provenance and peer review Not commissioned; externally peer reviewed.

Data availability statement Qualitative data from this study are available upon reasonable request by writing to the corresponding author.

Supplemental material This content has been supplied by the author(s). It has not been vetted by BMJ Publishing Group Limited (BMJ) and may not have been peer-reviewed. Any opinions or recommendations discussed are solely those of the author(s) and are not endorsed by BMJ. BMJ disclaims all liability and responsibility arising from any reliance placed on the content. Where the content includes any translated material, BMJ does not warrant the accuracy and reliability of the translations (including but not limited to local regulations, clinical guidelines, terminology, drug names and drug dosages), and is not responsible for any error and/or omissions arising from translation and adaptation or otherwise.

Open access This is an open access article distributed in accordance with the Creative Commons Attribution Non Commercial (CC BY-NC 4.0) license, which permits others to distribute, remix, adapt, build upon this work non-commercially, and license their derivative works on different terms, provided the original work is properly cited, appropriate credit is given, any changes made indicated, and the use is non-commercial. See: http://creativecommons.org/licenses/by-nc/4.0/.

ORCID iD

Negar Monazam Tabrizi http://orcid.org/0000-0003-1300-5030

\section{REFERENCES}

1 WHO. Patient safety fact file, patient safety and risk management service delivery and safety, 2019. Available: https://www.who.int/ features/factfiles/patient_safety/patient-safety-fact-file.pdf?ua $=1$ [Accessed $01 \mathrm{Apr}$ 2020].

2 WHO. World Health Organization's 10 facts on patient safety, 2018. Available: https://www.who.int/features/factfiles/patient_safety/en/ [Accessed 01 Apr 2020].

3 Hignett S, Lang A, Pickup L, et al. More holes than cheese. what prevents the delivery of effective, high quality and safe health care in England? Ergonomics 2018;61:5-14.

4 Dixon-Woods M, Baker R, Charles K, et al. Culture and behaviour in the English National health service: overview of lessons from a large multimethod study. BMJ Qual Saf 2014;23:106-15.

5 Waring J, Currie G, Crompton A, et al. An exploratory study of knowledge brokering in hospital settings: facilitating knowledge sharing and learning for patient safety? Soc Sci Med 2013;98:79-86.

6 Kim Yong-Mi, Newby-Bennett D, Song Hee-Joon. Knowledge sharing and institutionalism in the healthcare industry. J Knowl Manag 2012;16:480-94.

7 Currie G, Waring J, Finn R. The limits of knowledge management for UK public services modernization: the case of patient safety and service quality. Public Adm 2008;86:363-85.

8 Leape LL, Berwick DM. Five years after to err is human: what have we learned? JAMA 2005;293:2384-90.

9 Reason J. Human error: models and management. BMJ 2000;320:768-70.

10 Evans SM, Berry JG, Smith BJ, et al. Attitudes and barriers to incident reporting: a collaborative hospital study. Qual Saf Health Care 2006;15:39-43. 
11 Reason J. Managing the risks of organizational accidents. Routledge, 2016.

12 Zhao B. Learning from errors: the role of context, emotion, and personality. J Organ Behav 2011;32:435-63.

13 Donaldson MS, Corrigan JM, Kohn LT. To err is human: building a safer health system. Vol 6. National Academies Press, 2000.

14 Sitkin SB. Learning through failure: the strategy of small losses. In Staw BM, ed. Research in organizational behavior. Greenwich, CT: JAI Press, 1992: vol. 14. 231-66.

15 Khanna R, Guler I, Nerkar A. Fail often, fail big, and fail fast? learning from small failures and R\&D performance in the pharmaceutical industry. Acad Manag J 2016;59:436-59.

16 Stead W, Lin H. Computational technology for effective healthcare: immediate steps and strategic directions. Washington, DC: The National Academic Press, 2009.

17 Department of Health. An organisation with a memory report of an expert group learning from adverse events in the NHS. London: The Stationery Office, 2000.

18 Vincent C, Stanhope N, Crowley-Murphy M. Reasons for not reporting adverse incidents: an empirical study. J Eval Clin Pract 1999:5:13-21.

19 Archer S, Hull L, Soukup T, et al. Development of a theoretical framework of factors affecting patient safety incident reporting: a theoretical review of the literature. BMJ Open 2017;7:1-16.

20 Lawton R, Parker D. Barriers to incident reporting in a healthcare system. Qual Saf Health Care 2002;11:15-18.

21 Waring JJ. Beyond blame: cultural barriers to medical incident reporting. Soc Sci Med 2005;60:1927-35.

22 Tucker AL, Nembhard IM, Edmondson AC. Implementing new practices: an empirical study of organizational learning in hospital intensive care units. Manage Sci 2007;53:894-907.

23 McKee L, Charles K, Dixon-Woods M, et al. 'New' and distributed leadership in quality and safety in health care, or 'old' and hierarchical? an interview study with strategic stakeholders. J Health Serv Res Policy 2013;18:11-19.

24 Heugens PPMAR, Lander MW, Agency! Structure!. Structure! agency! (and other Quarrels): a meta-analysis of institutional theories of organization. Acad Manage J 2009;52:61-85.

25 Edmondson AC. Learning from failure in health care: frequent opportunities, pervasive barriers. Qual Saf Health Care 2004;13 Suppl 2:ii3-9.

26 Beasley JW, Escoto KH, Karsh B-T. Design elements for a primary care medical error reporting system. WMJ 2004;103:56-9.

27 Kankanhalli A, Tanudidjaja F, Sutanto J, et al. The role of it in successful knowledge management initiatives. Commun ACM 2003;46:69-73

28 Hendriks P. Why share knowledge? the influence of ICT on the motivation for knowledge sharing. Knowl. Process Manag 1999;6:91-100.

29 Wang CL, Ahmed PK. Organisational learning: a critical review. The Learning Organization 2003;10:8-17.

30 Barach P, Small SD. Reporting and preventing medical mishaps: lessons from non-medical near miss reporting systems. BMJ 2000;320:759-63.

31 Braithwaite J, Westbrook MT, Travaglia JF, et al. Cultural and associated enablers of, and barriers to, adverse incident reporting. Qual Saf Health Care 2010;19:229-33.

32 Department of Health. Building a Safe NHS for Patients. London: The Stationery Office, 2001.
33 The Guardian. 750 avoidable deaths a month in NHS hospitals, study finds, 2015. Available: https://www.theguardian.com/society/2015/ jul/14/avoidable-deaths-nhs-hospitals-study

34 Makary MA, Daniel M. Medical error-the third leading cause of death in the US. BMJ 2016;353:i2139.

35 Drupsteen L, Groeneweg J, Zwetsloot GIJM. Critical steps in learning from incidents: using learning potential in the process from reporting an incident to accident prevention. Int J Occup Saf Ergon 2013;19:63-77.

36 Akselsson R, Jacobsson A, Bötjesson M, et al. Efficient and effective learning for safety from incidents. Work 2012;41 Suppl 1:3216-22.

37 Jacobsson A, Ek Åsa, Akselsson R. Learning from incidents - a method for assessing the effectiveness of the learning cycle. $J$ Loss Prev Process Ind 2012;25:561-70.

38 Sujan MA, Huang $\mathrm{H}$, Braithwaite J. Learning from incidents in health care: critique from a safety-II perspective. Saf Sci 2017;99:115-21.

39 National Audit Office. A short guide to the Department of health and NHS England department of health, 2017. Available: https://www. nao.org.uk/wp-content/uploads/2017/09/2017-Short-Guide-to-theDepartment-of-Health-and-NHS-England.pdf [Accessed 04 June 2021].

40 Madsen PM, Desai V. Failing to learn? the effects of failure and success on organizational learning in the global orbital Launch vehicle industry. Acad Manage J 2010;53:451-76.

41 Edmondson AC. Strategies of learning from failure. Harv Bus Rev 2011;89:48-55

42 Miles MB, A.M H. Analyzing qualitative data: a sourcebook of new methods. Center for Policy Research, 1983. https://us.sagepub.com/ en-us/nam/qualitative-data-analysis/book246128

43 Saunders M, Lewis P, Thornhill A. Research methods for business students. 7th edn. Financial Times Prentice Hall, 2017.

44 Myers MD, Newman M. The qualitative interview in is research: examining the craft. Information and Organization 2007;17:2-26.

45 Fisher RJ. Social desirability bias and the validity of indirect Questioning. J Consum Res 1993;20:303-15.

46 Myers MD. Qualitative research in business and management. London: Sage, 2009.

47 Braun V, Clarke V. Using thematic analysis in psychology. Qual Res Psychol 2006;3:77-101.

48 Helmreich RL, Merritt AC. Culture at work in aviation and medicine: national, organizational and professional influences. Routledge, 2019.

49 Sujan M. An organisation without a memory: a qualitative study of hospital staff perceptions on reporting and organisational learning for patient safety. Reliab Eng Syst Saf 2015;144:45-52.

50 Adler PS. Market, hierarchy, and trust: the knowledge economy and the future of capitalism. Organization Science 2001;12:215-34.

51 Argyris C, Schön DA. Organisational learning II: theory, method and practice. MA: Addison-Wesley, Reading, 1996.

52 Sujan MA, Embrey D, Huang $H$. On the application of human reliability analysis in healthcare: opportunities and challenges. Reliab Eng Syst Saf 2020;194:106189.

53 Birken SA, Lee S-YD, Weiner BJ. Uncovering middle managers' role in healthcare innovation implementation. Implementation Science 2012;7:1-12.

54 WHO. Patient safety: global action on patient safety: report by the director-general, 2018. Available: https://apps.who.int/gb/ebwha/ pdf_files/WHA72/A72_R6-en.pdf?ua=1 [Accessed 20 Aug 2020]. 